\title{
MICROCHARACTERIZATION OF INTERFACES
}

\section{P. M. PETROFF}

Bell Laboratories, 600 Mountain Avenue, Murray Hill, NJ07974 (U.S.A.)

The atomic structure of semiconductor heterostructure interfaces and metalsemiconductor interfaces are best characterized by transmission electron microscopy (TEM). Both phase contrast TEM and structure factor contrast TEM are able to distinguish very small structural (two monolayers) or compositional (less than $0.2 \%$ ) fluctuations at interfaces. Applications of these techniques to the study of the roughening transition temperature at the $\mathrm{Ga}_{1-x} \mathrm{Al}_{x} \mathrm{As}-\mathrm{GaAs}$ and $\mathrm{Ga}_{1-x} \mathrm{Al}_{x} \mathrm{As}-\mathrm{Ge}$ interfaces grown by molecular beam epitaxy are presented. Minority carrier recombination at interfaces is characterized on a microscopic scale by low temperature cathodoluminescence. This technique is used to demonstrate the role of interfaces in gettering defects in $\mathrm{Ga}_{1-x} \mathrm{Al}_{x} \mathrm{As} / \mathrm{GaAs}$ heterostructures. Finally, the effects of interfacial strain in producing a localization of the luminescence in GaAs quantum well wire structures will be discussed. 